\title{
Laparoscopic Resection and Pre-Operative Imaging of Primary Retroperitoneal Mucinous Neoplasms: A Retrospective Case Series
}

This article was published in the following Dove Press journal: Cancer Management and Research

\author{
Yuyang Zhang' \\ Jiejin Yang $\mathbb{D D}^{2}$ \\ Zeyang Chen' \\ Jiali Sun ${ }^{2}$ \\ Pengyuan Wang' \\ 'Department of General Surgery, Peking \\ University First Hospital, Peking \\ University, Beijing, People's Republic of \\ China; ${ }^{2}$ Department of Radiology, Peking \\ University First Hospital, Peking \\ University, Beijing, People's Republic of \\ China
}

Purpose: The purpose of this paper is to present the clinicopathological features of primary retroperitoneal mucinous neoplasms (PRMNs), to evaluate the diagnostic role of preoperative radiological examinations, and to determine the feasibility of total resection using the laparoscopic approach.

Methods: We retrospectively analysed the clinicopathological and radiological features of 10 PRMN cases who underwent surgical resection from January 2000 to December 2019.

Results: Ten PRMN cases were evaluated in this study, including 1 malignant case, 4 borderline cases, and 5 benign cases. The most common primary complaints were pain $(60 \%)$ and palpable mass $(60 \%)$. Of the 8 cases with pre-operative CT results, $75 \%$ of the borderline or malignant cysts and none of the benign cysts were large in size (size $>11.6 \mathrm{~cm})$. Half of the non-benign masses and none of the benign ones were lobulated. Thick wall ( $\geq 3 \mathrm{~mm}$ ) and irregular wall were found in $25 \%$ and $50 \%$ of the non-benign cases, respectively. Wall enhancement was detected in $75 \%$ of the non-benign cases and $25 \%$ of the benign cases. Enhanced mural nodules were identified in $50 \%$ of the non-benign patients. Septa were found in $25 \%$ of the non-benign cases. Complete dissection of the tumour without rupture or post-operative complications was achieved laparoscopically in $90 \%$ of the cases. All patients were alive with no recurrence at the last follow-up at an average of $28.1 \pm 26.6$ months.

Conclusion: Larger size and lobulation of the cyst, presence and contrast enhancement of thick and irregular cyst walls, mural nodules, and internal septa may be imaging features suggestive of malignancy. Complete resection of PRMN through the laparoscopic approach is feasible in experienced institutions.

Keywords: primary retroperitoneal mucinous tumour, laparoscopy, radiological features, CT

\section{Introduction}

Primary retroperitoneal mucinous neoplasm (PRMN) is extremely rare, with a prevalence of less than $2 \%$ among all resected retroperitoneal masses. ${ }^{1}$ Histologically, it is similar to its ovarian counterparts and can be divided into 3 categories: primary retroperitoneal mucinous cystadenoma (PRMC), borderline primary retroperitoneal mucinous cystadenoma (bPRMC), and primary retroperitoneal mucinous cystadenocarcinoma (PRMCa). PRMN most often occurs in perimenopausal females, the aetiology of which is still unknown. ${ }^{2}$ Clinical evaluations, ranging from patient symptoms and laboratory examinations to biopsy or imaging-guided cyst aspiration, often provide limited evidence for an accurate diagnosis. ${ }^{2,3}$ Therefore, pre-operative
Correspondence: Pengyuan Wang Department of General Surgery, Peking University First Hospital, Peking

University, Beijing I00034, People's

Republic of China

Tel +86-|39||I 58750

Fax +86-10-83572413

Email wangpengyuanpku@I63.com 
imaging studies are of particular significance. The mainstay treatment is complete surgical resection, as is the case with most retroperitoneal tumours. Although long-term prognosis is excellent for patients with PRMC and bPRMC, patients with PRMCa have an overall 5-year survival rate of only $68-75 \%$ and a recurrence-free survival rate of only $45-60 \%$. $^{2,3}$ Thus, when operating on cysts with potential malignancy based on pre-operative radiological studies, special precautions should be taken due to the possibility of tumour dissemination. In total, 153 PRMN cases have been published since 1924; however, the predominance of single case reports and small case series, aside from one case series of 18 patients, has made consensus building in PRMN management unrealistic. $^{4-13}$ In this study, we report the largest series of laparoscopically resected PRMNs to date, as well as the second-largest case series of PRMNs as a whole. A case example is given to illustrate the typical clinical course with a successful pre-operative radiological diagnosis. With an emphasis on the importance of pre-operative imaging and the feasibility of the laparoscopic approach, this retrospective study aims to enhance understanding and improve treatment and outcomes for this rare disease.

\section{Patients and Methods}

\section{Patient Selection}

The clinical database of Peking University First Hospital (PKUFH) was searched and 10 cases of PRMN who underwent surgical treatment at PKUFH from January 2000 to December 2019 were identified, including 1 case of PRMCa, 4 cases of bPRMC, and 5 cases of PRMC. As there is currently no appropriate way to diagnose PRMN preoperatively based on clinical features, imaging, or biopsy, the diagnosis criteria we adopted in this study were pathological. $^{1,14}$ Specifically, diagnosis of PRMC was based on the presence of a single layer of columnar cells with abundant intracellular mucin and small basilar nuclei lines in the cyst. bPRMC was defined as a proliferating noninvasive neoplasm with more than minor foci of nuclear atypia of slight-to-moderate degree. Loss of polarity and multilayering of neoplastic cells were also commonly found in bPRMCs. bPRMC with intraepithelial carcinoma were characterized by marked atypia without stromal invasion. ${ }^{5}$ Detailed definition of stromal invasion was provided by Riopel et al. ${ }^{15}$ Tumours were classified as PRMCa if stromal invasion greater than $3 \mathrm{~mm}$ at the maximum dimension or larger than $10 \mathrm{~mm}^{2}$ in area was present. ${ }^{16}$ Aside from pathological identification, to be included in the series, histologic, imaging, or intraoperative evidence that excluded organ involvement, relevant ovarian lesions, as well as metastatic origin was necessary. Non-neoplastic lesions and masses with unclear or unavailable pathologies were excluded from the analysis.

\section{Data Collection}

Clinical information was gathered mainly from the electronic medical record (EMR) system of PKUFH. Relevant surgeons and clinical oncologists were consulted without knowing the aim of the study if certain surgical and oncological information were deemed ambiguous or inadequate. Survival and prognosis data were confirmed and, in some cases, renewed via telephone follow-up throughout February 2020. Clinical features were recorded as follows: patient's age at diagnosis, sex, symptoms on presentation, surgical approach and compliance with tumour-free principles, gross features of the resected tumour, and followup status.

Pathological data were collected from the EMR system of PKUFH with the assistance of senior pathologists who kindly reviewed indefinite or disputable results. Immunohistochemical staining results were available in 3 cases and were recorded accordingly.

Radiological features were acquired from PKUFH's EMR system. The original pre-operative contrast-enhanced abdominal computed tomography (CT) films were extracted within 1 month from our institution's imaging system for further imaging analysis, as described below.

\section{CT Imaging Analysis}

Original pre-operative CT exams were retrospectively evaluated by two senior radiologists who were blinded to the patient's clinicopathological data and previous imaging. If there were differences of opinion between the two reviewers, consensus was reached through reevaluation and negotiation. The following tumour characteristics on CT were collected for analysis: location, size, shape, margin, presence of thick wall, mural nodules, internal septa, calcification, and evidence of lymphadenopathy and ascites.

The size of the lesion, as indicated by the maximal crosssectional diameter, was measured by digital callipers; a diameter above average was considered a large cyst. The shape of the tumour was classified as either round or lobulated. Tumour margins were categorised as either welldefined, or, when the tumour infiltrated neighbouring tissues or had a blurred border, as ill-defined. When the thickness of 
a cyst wall, determined by measuring the maximum thickness of the wall, was no less than $3 \mathrm{~mm}$, it was categorised as a thick wall. A mural nodule was defined as a solid lesion protruding into the mass from the cyst wall. The size of the mural nodule was measured by height and width. Height refers to the maximal vertical length from the outer surface of the cyst wall to the top of the nodule, while width refers to the maximum perpendicular length to the height. The growth patterns of the nodules were categorised as either broadbased or polypoid. When internal septa were present, the maximum thickness was recorded. Contrast enhancement of the lesion was evaluated with a focus on the cyst wall, mural nodules, and internal septa. Regions of calcification were defined as high-attenuation foci. The presence of lymph nodes greater than $1 \mathrm{~cm}$ in the shortest axis was considered lymphadenopathy.

\section{Results}

\section{Cases Series}

Among the 519 cases of retroperitoneal neoplasms resected over the past 20 years, we identified 10 cases fulfilling the aforementioned diagnostic criteria for PRMN. Aside from PRMNs, the remaining cases comprised 189 mesenchymal tumours, 137 neurogenic tumours, 105 secondary malignancies, including lymphatic metastasis, 24 germ cell neoplasms, 16 lymphoproliferative diseases, and 38 miscellaneous neoplastic pathologies.
The estimated prevalence of PRMN among the identified retroperitoneal neoplasms was $1.96 \%$.

A summary of the clinicopathologic features of the 10 cases is provided in Table 1. All patients were female with an average age of $38.0 \pm 15.6$ years (range, 15-65 years). The two most common primary complaints were abdominal pain $(60 \%)$ and palpable mass $(60 \%)$, while only 1 patient was asymptomatic. The results of serum tumour marker analysis were available for 5 of the 10 patients (50\%). Carbohydrate antigen 125 (CA 125) was slightly elevated in 2 of 4 cases, with a serum value of $42.3 \mathrm{IU} / \mathrm{mL}$ and $47.5 \mathrm{IU} / \mathrm{mL}$ in 1 malignant and 1 borderline case, respectively. Carcinoembryonic antigen (CEA) and CA199 were checked and were normal in 5 cases. Pathological classifications were as follows: 1 case of PRMCa $(1 / 10,10 \%)$ with necrosis, 4 cases of bPRMC $(4 / 10,40 \%)$, including 1 with intraepithelial carcinoma and 1 with focal carcinoma, and 5 cases of PRMC (5/10, $50 \%$ ). All masses were found in the lateral retroperitoneal space. Serous components were present in 1 case each of PRMCa and bPRMC.

Among the various imaging modalities, abdominal ultrasonography and CT were the two most frequently used radiological investigations. Magnetic resonance imaging (MRI) was adopted in 2 of 10 cases (20\%) and had provided equivalent, if not less, information as provided by the parallel CT exam. Overall, 8 of 10 patients $(80 \%)$ underwent CT scans, the characteristics of which are

Table I Clinicopathological Features of 10 Cases of PRMN

\begin{tabular}{|l|l|l|l|l|l|l|l|l|}
\hline Case & Diagnosis & $\begin{array}{l}\text { Age } \\
\text { (Years) }\end{array}$ & Sex & Symptom & Side & $\begin{array}{l}\text { Mass } \\
\text { Removal }\end{array}$ & Gross Features & Follow-Up \\
\hline I & $\begin{array}{l}\text { Mucinous and serous } \\
\text { PRMCa }\end{array}$ & 37 & F & Pain, mass & Rt & LC, TR, TF & $\begin{array}{l}\text { Cystic-solid mass with fish-meat like appearance, gray } \\
\text { viscous fluid }\end{array}$ & NED, 60m \\
\hline 2 & PRMC & I5 & F & Pain, mass & Rt & LC, TR, TF & Cyst, clear fluid & $/$ \\
\hline 3 & bPRMC & 65 & F & Mass & Rt & LC, TR, TF & Partially septated cyst & NED, 6m \\
\hline 4 & PRMC & 58 & F & Pain & Rt & LC, TR, TF & Small cyst & NED, 74m \\
\hline 5 & $\begin{array}{l}\text { Mucinous and serous } \\
\text { bPRMC }\end{array}$ & 23 & F & Mass & Rt & LC, TR, TF & Unilocular cyst & $/$ \\
\hline 6 & PRMC & 26 & F & Pain & Lt & LC, TR, TF & Soft cyst & NED, I3m \\
\hline 7 & PRMCa in bPRMC & 45 & F & Mass & Rt & LC, TR, TF & Cyst & NED, 8m \\
\hline 8 & PRMC & 29 & F & None & Rt & LC, TR, TF & Cyst & NED, 3Im \\
\hline 9 & PRMC & 38 & F & Pain & Lt & LC, TR, TF & Cyst & NED, 3Im \\
\hline 10 & bPRMC with ICa & 44 & F & Pain, mass & Lt & LC, TR, not & Cyst with solid components & NED, 2m \\
\hline
\end{tabular}


summarised in Table 2. The average cyst size based on CT results was $11.6 \pm 8.0 \mathrm{~cm}$ (range, 2.4-27.0). Cysts with a diameter above average were considered to be large cysts.

The CT features that might be helpful for differential diagnosis are summarised in Figure 1. None of the benign tumours and 3 of 4 borderline or malignant tumours $(75 \%)$ were considered large cysts. Further, 2 of 4 non-benign masses $(50 \%)$ and none of the benign masses were lobulated. A thick cyst wall of no less than $3 \mathrm{~mm}$ was found in 1 borderline case $(1 / 4,25 \%)$ with intraepithelial carcinoma, but none of the benign cases. Irregular or nonsmooth walls were only found in 2 of 4 borderline or malignant cysts (50\%). Wall enhancement was detected in 3 of 4 borderline or malignant cases $(75 \%)$ and 1 of 4 benign cases $(25 \%)$. Enhanced mural nodules were identified in 2 of 4 borderline or malignant patients (50\%) and none of the benign cases. The nodules were broad-based with a mean height of $3.2 \pm 2.0 \mathrm{~cm}$ and a mean width of 1.7 $\pm 0.3 \mathrm{~cm}$. Internal septa of $1 \mathrm{~mm}$ were detected in only 1 of 4 borderline cases (25\%) with focal carcinoma. Calcification was present in 2 of 4 benign cases $(50 \%)$ and 1 of 4 non-benign ones (25\%). No lymphadenopathy was observed. Ascites were found in half of the benign (2/ $4,50 \%)$ and borderline or malignant cases $(2 / 4,50 \%)$, respectively. Although retroperitoneal position was correctly localised by $\mathrm{CT}$ in all cases, accurate diagnosis of PRMN was obtained in only 2 of 10 recent patients $(20 \%)$. Imaging-based misdiagnosis included: intestinal duplication, epidermoid cyst, pseudomyxoma, appendiceal mucocele, lymphangioma, mesenteric cyst, teratoma, and neurogenic tumour.

Complete resection of the tumour without rupture was achieved laparoscopically in 9 of 10 cases (90\%); however, in the typical case presented below, the tumour-free principles were not strictly complied with due to cyst decompression outside the retrieval bag. The mean tumour size based on post-operative gross features was 12.3 $\pm 6.7 \mathrm{~cm}$ (range, $3-25 \mathrm{~cm}$ ). Among the 9 laparoscopically resected cases, the average operative time was $79.7 \pm 38.5$ min, the estimated blood loss was $37.8 \pm 18.6 \mathrm{~mL}$, and the post-surgical hospital length of stay was $2.7 \pm 0.9$ nights. There were no procedure-related complications or conversions to laparotomy.

Immunohistochemistry staining results were available in 3 of 10 cases (30\%). Cytokeratin 7 (CK 7) was strongly positive in all 3 cases in which it was evaluated, while CK 20 was only partially positive in 1 of 3 patients (33\%). CA
Table 2 CT Features of 8 Cases of PRMN

\begin{tabular}{|c|c|c|}
\hline \multirow[t]{2}{*}{ CT Features } & \multicolumn{2}{|c|}{ Pathology (N/\%) } \\
\hline & $\begin{array}{l}\text { Benign } \\
(4 / 50)\end{array}$ & $\begin{array}{l}\text { Borderline or Malignant } \\
(4 / 50)\end{array}$ \\
\hline \multicolumn{3}{|l|}{ Size } \\
\hline$\geq 11.6 \mathrm{~cm}$ & $0(0)$ & $3(75)$ \\
\hline \multicolumn{3}{|l|}{ Shape } \\
\hline Round & $4(100)$ & $2(50)$ \\
\hline Lobulated & $0(0)$ & $2(50)$ \\
\hline \multicolumn{3}{|l|}{ Margin } \\
\hline Well-defined & $4(100)$ & $4(100)$ \\
\hline III-defined & $0(0)$ & $0(0)$ \\
\hline \multicolumn{3}{|l|}{ Cyst wall } \\
\hline Thick wall ( $\geq 3 \mathrm{~mm})$ & $0(0)$ & I (25) \\
\hline Irregular wall & $0(0)$ & $2(50)$ \\
\hline Contrast enhancement & I (25) & $3(75)$ \\
\hline \multicolumn{3}{|l|}{ Mural nodules } \\
\hline Presence & $0(0)$ & $2(50)$ \\
\hline \multicolumn{3}{|l|}{ Size $(\mathrm{cm})$} \\
\hline Height & I & $3.2 \pm 2.0$ \\
\hline Width & I & $1.7 \pm 0.3$ \\
\hline \multicolumn{3}{|l|}{ Growth pattern } \\
\hline Broad-based & I & $2(50)$ \\
\hline Polypoid & I & $0(0)$ \\
\hline Contrast enhancement & I & $2(50)$ \\
\hline \multicolumn{3}{|l|}{ Internal septa } \\
\hline Presence & $0(0)$ & I (25) \\
\hline Thickness (mm) & I & 1 \\
\hline Contrast enhancement & I & I (25) \\
\hline Calcification & $2(50)$ & I (25) \\
\hline Lymphadenopathy & $0(0)$ & $0(0)$ \\
\hline Ascites & $2(50)$ & $2(50)$ \\
\hline
\end{tabular}

Abbreviations: bPRMC, borderline primary retroperitoneal mucinous cystadenoma; CA, carbohydrate antigen; CEA, carcinoembryonic antigen; CK, cytokeratin; $\mathrm{CT}$, computed tomography; EMR, electronic medical record; ER/PR, oestrogen and progesterone receptors; LC, laparoscopy; Lt, left; MCNs, mucinous cystic neoplasms; MRI, magnetic resonance imaging; PET/CT, positron emission tomography/ computed tomography; PKUFH, Peking University First Hospital; PRMC, primary retroperitoneal mucinous cystadenoma; PRMCa, primary retroperitoneal mucinous cystadenocarcinoma; PRMN, primary retroperitoneal mucinous neoplasm; Rt, right; SCNs, serous cystic neoplasms; TF, tumor-free; TR, total resection.

125 was assessed and stained positive in 1 PRMC case. CDX-2 was stained in 2 cases and both were negative. Oestrogen and progesterone receptors (ER/PR) were negative in 1 case of bPRMC with intraepithelial carcinoma.

With respect to adjuvant chemotherapy, only 1 patient whose pathology was mucinous and serous cystadenocarcinoma with necrosis received 3 cycles of paclitaxel $\left(135 \mathrm{mg} / \mathrm{m}^{2}\right)$ and cisplatin $\left(70 \mathrm{mg} / \mathrm{m}^{2}\right)$. The patient showed no evidence of disease during her last follow-up at 60 months. 


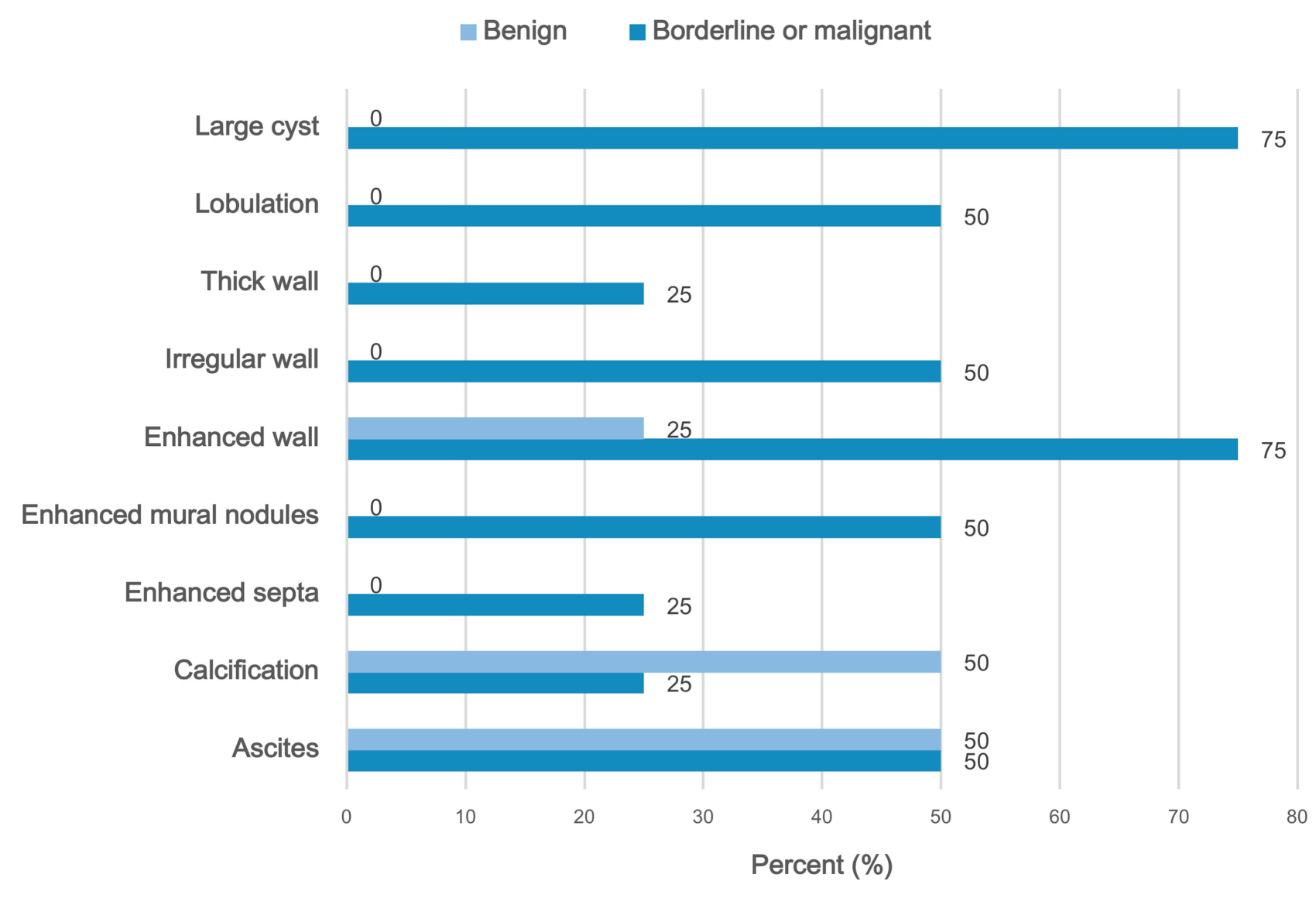

Figure I CT features for differential diagnosis between benign PRMNs and borderline or malignant PRMNs.

The general prognosis of PRMN is promising. Among 8 cases with an average length of follow-up of $28.1 \pm 26.6$ months (range, 2-74 months), including 2 borderline cases with focal carcinoma or intraepithelial carcinoma, all patients were alive with no recurrence at last follow-up.

\section{A Typical Case (Case 10)}

A 44-year-old female with no remarkable medical history was admitted to hospital complaining of abdominal distention and a growing palpable mass for more than a year. On physical examination, a smooth football-sized mass was palpable in the left lower quadrant with mild tenderness. Abdominal ultrasonography and contrast-enhanced CT showed a large cystic mass with solid components. Further consultation on CT results indicated the presence of an enhanced mural nodule of $4.6 \times 1.5 \mathrm{~cm}$ and an enhanced thick irregular wall of $6 \mathrm{~mm}$ (Figure 2). Consequently, an imaging diagnosis of retroperitoneal cystadenocarcinoma was made. Laboratory findings were within normal limits. Laparoscopic surgery was then performed, revealing an ovalshaped high-tension cyst located in the left retroperitoneal space, pushing inwards on the descending and sigmoid colons. After effortless separation from adjacent tissues, $1000 \mathrm{~mL}$ of muddy brown liquid was drained through a puncture hole of $0.3 \mathrm{~cm}$, followed by careful suture of the cyst wall. The huge mass was then completely removed; a specimen retrieval bag was used to retrieve the tumour. However, since the initial decompression of the mass was not protected by the retrieval bag, although no gross spillage of fluid content was visible, the possibility of intraperitoneal micrometastasis could not be ruled out.

On gross examination, the tumour was $20 \mathrm{~cm}$ in diameter and was not connected to the left ovary. The cyst had a thick and rough wall with mural nodules of different sizes and solid components. Microscopically, mucinous and papillary glands with focal areas of haemorrhage and interstitial fibrosis were present. There was mild to moderate epithelial atypia with areas of intraepithelial carcinoma represented by severe nuclear atypia. Immunohistochemistry exam showed diffuse positive staining for CK 7 and CEA, as well as focal positive staining for CK 20 (Figure 3). Considering the retroperitoneal location of the mass, the 

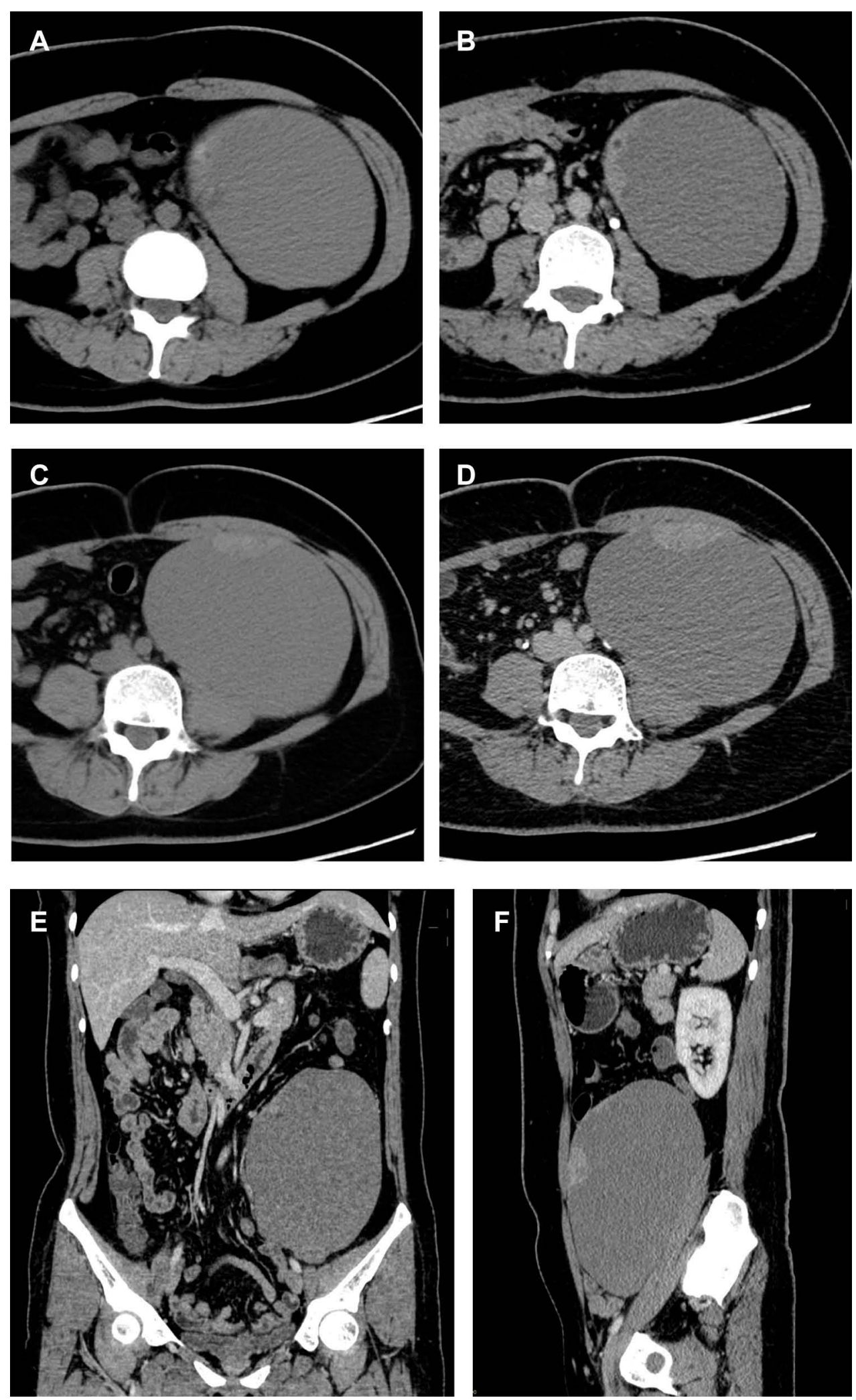

Figure 2 Pre-operative imaging of a 44-year-old female with intraepithelial carcinoma in bPRMC (Case 10). Unenhanced and enhanced axial CT scan (A and B) reveals an oval-shaped, well-circumscribed cyst with a thick irregular wall and contrast enhancement in the venous phase, while axial CT scan (C and $\mathbf{D})$ shows a broad-based mural nodule on the front wall with contrast enhancement in the venous phase. CT scan in the coronal $(\mathbf{E})$ and sagittal $(\mathbf{F})$ planes also demonstrates an irregular cyst wall and mural nodules in the venous phase. 

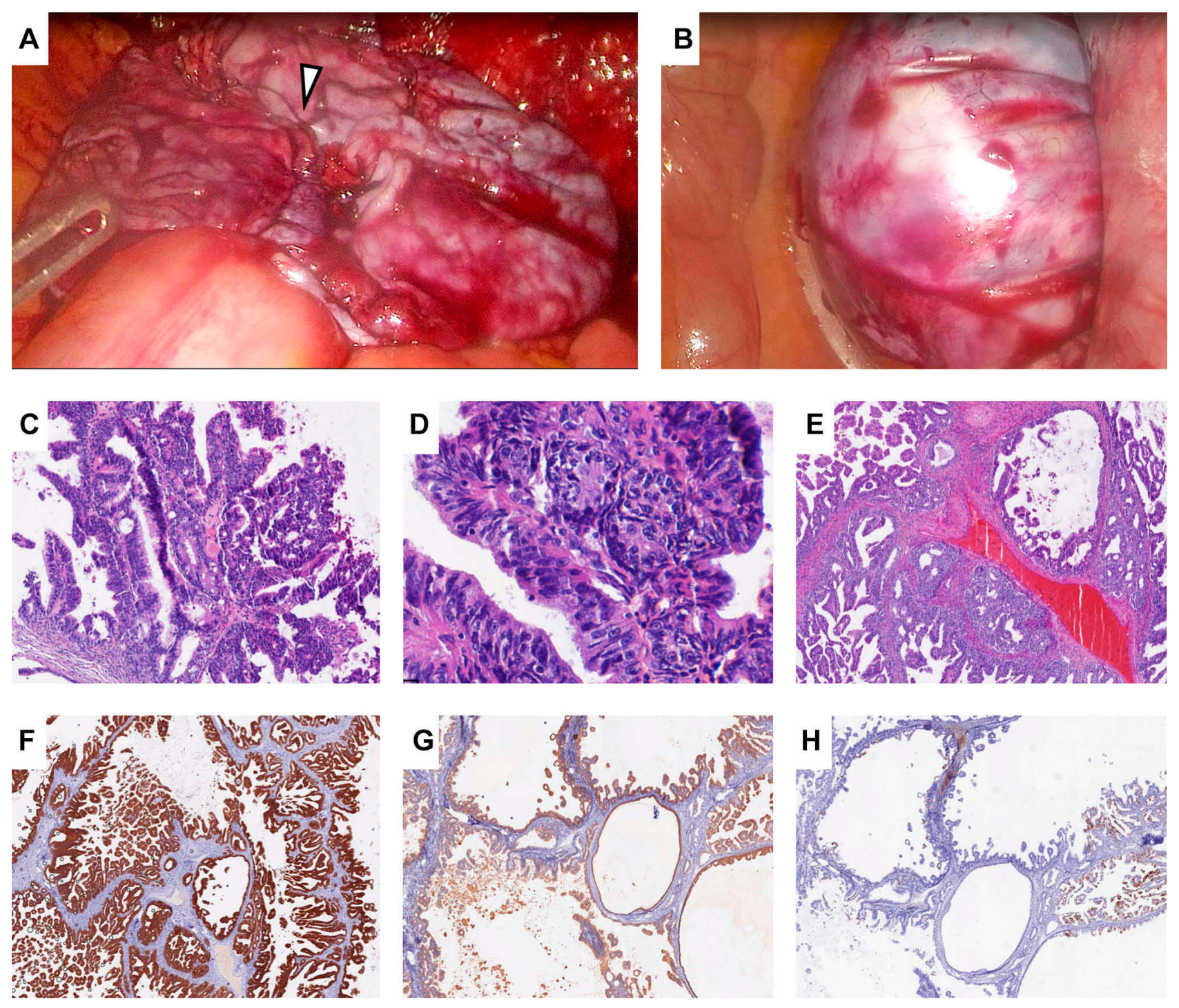

Figure 3 Surgical highlights and microscopic features of a 44-year-old female with intraepithelial carcinoma in bPRMC (Case 10). The huge cyst was drained laparoscopically and the puncture hole (indicated by the white triangle) was carefully sutured (A). The mass was then put into a specimen bag and was taken out through a trocar hole (B). Hematoxylin-eosin sections show areas of intraepithelial carcinoma marked by severe nuclear atypia (C and $\mathbf{D})$ with haemorrhage and interstitial fibrosis (E). Immunohistochemistry stains show diffuse immunoreaction for CK 7 (F) and CEA (G), as well as focally positive staining for CK $20(\mathbf{H})$.

tumour was therefore diagnosed as bPRMC with intraepithelial carcinoma.

The patient's recovery was uneventful, and she was discharged on the fifth post-operative day. Although no further treatment was given, post-operative gastroscopy, colonoscopy, and positron emission tomography/computed tomography (PET/CT) were conducted to rule out metastatic disease. The patient had no evidence of disease 2 months after surgery.

\section{Discussion}

PRMN is a rare disease. There are only 153 published cases to date, most of which are single case reports of varied quality or small case series of no more than 3 patients. ${ }^{2,17-20}$ The estimated prevalence of PRMN among retroperitoneal neoplasms resected in our institution is $1.96 \%$. One systematic review and one meta-analysis of PRMCa, and one meta-analysis of PRMN have presented general information on epidemiology and clinical features; the results of these prior studies are consistent with our study. ${ }^{2,3,21}$ In previous studies, serum tumour markers such as CEA, CA 125, and CA 199 were reportedly elevated in a small portion of patients, but it has been demonstrated that they have neither diagnostic nor prognostic significance, as is the case with pre-operative biopsy. ${ }^{2,3}$ In our series, not surprisingly, these aforementioned diagnostic tools exhibited poor diagnostic efficacies. 
As for pre-operative imaging, according to published cases and reviews, there are no identified pathognomonic features; hence, precise diagnosis without the guidance of pathology reports is normally difficult. ${ }^{2}$ Nevertheless, based on the accumulated experience in our institution over the past 20 years, we would like to attach a more significant role to radiologists and pre-operative imaging. In fact, we found that larger size and lobulation of the cyst, presence and contrast enhancement of thick and irregular cyst walls, mural nodules, and internal septa might help identify a group of tumours with a more malignant nature. Indeed, intramural nodules of any nature were found to be correlated with malignancy in PRMN in a recent metaanalysis. ${ }^{2}$ Moreover, it was reported that certain imaging features were more suggestive of a benign mass, including thin wall, lack of internal structures or contrast enhancement, and no evidence of invasions such as peritoneal metastasis. ${ }^{18}$ Similarly, in terms of a retroperitoneal organ with more published studies, it has been reported that pancreatic mucinous cystic neoplasms (MCNs) are more likely to have enhanced cyst walls compared to serous cystic neoplasms (SCNs), and the extent of wall enhancement is higher in MCNs with malignancy than in benign ones. ${ }^{22,23}$

With regard to imaging modality, although ultrasonography generally stands out because of its low-cost advantage and universal availability, the drawback of its ambiguity compared to $\mathrm{CT}$ and MRI is much more prominent in the case of retroperitoneal masses. Further, in 2 cases with MRI results, the MRI results were not superior to the CT exams in terms of diagnostic significance. This seems to be in disagreement with a recent meta-analysis indicating that MRI might provide more detail about the surrounding soft tissue structures and information on internal structures with gadolinium enhancement. ${ }^{18}$ This discrepancy in findings may, to some extent, be due to contrast enhancement was not conducted in either of the cases in our series. In fact, in the case of retroperitoneal neoplasms, it is believed that both CT and MRI can reveal important features of tumours. ${ }^{24}$ Moreover, it is worth noting that accurate diagnosis of PRMN was successfully achieved in two recent consecutive patients, one of which was assisted by radiologic consultation. This improvement in diagnostic capacity might be explained by radiologists' growing familiarity and experience with this rare disease, which has been further augmented through various forms of collaboration, such as consultation.
All 10 patients in our series underwent total surgical tumour resection, which is considered the mainstay treatment. Regarding the surgical approach, almost all reported cases, except for 18 procedures, have chosen traditional laparotomy given the general large volume of the tumour. Nevertheless, with the maturity of modern surgical techniques and the increasing use of retrieval bags or endobags, laparoscopic excision, which can minimise hospital stay and deliver a quicker return to daily activities, deserves broader application among experienced surgeons. ${ }^{1,18,19}$ In our series, $90 \%(9 / 10)$ of the tumours, with an average diameter of $12.3 \pm 6.7 \mathrm{~cm}$ based on post-operative gross features, were completely resected laparoscopically. The small amount of blood loss, short operative time, and absence of major surgical complications collectively demonstrate the safety and feasibility of the laparoscopic approach. Furthermore, rapid recovery was achieved through laparoscopy, with an average post-surgical length of hospital stay of less than 3 nights. Tumour-free principles were strictly followed in most of the procedures through careful puncture and drainage within retrieval bags or endobags, without spillage; the one exception is the typical case presented above, which had a risk of intra-abdominal micrometastasis. In addition, CK 7 was diffusely positive in all cases (3/3) and CK 20 was focally positive in 33\% cases $(1 / 3)$. The immunoreactive pattern was not different from mucinous tumours derived from the ovaries or pancreas; therefore, the significance of immunohistochemical investigation for differential diagnosis remains to be investigated. To our knowledge, this is the largest series of laparoscopically resected PRMNs reported in the literature to date.

The need for adjuvant therapy and the standard regime remains an area in need of clarification. Combinations of cyclophosmide, adriamycin, cisplatin, carboplatin, and paclitaxel have been explored in a few cases with highrisk features. ${ }^{1,3,17}$ In our series, where most cases had no intra-operative rupture, pathology of aggressive nature, or evidence of recurrence, adjuvant chemotherapy with paclitaxel and cisplatin was adopted in only 1 patient with PRMCa, whose last follow-up after 60 months showed no evidence of recurrence. Due to the inadequate number of patients and the inert nature of the disease, no conclusions could be drawn with regard to the efficacy of adjuvant chemotherapy or any specific regimen. Indeed, no recurrence was identified in any case in our series, with an average follow-up of 28.1 \pm 26.6 months (range, 2-74 months). Survival analysis could not be carried out because 
of the small sample sizes, lack of long-term follow-up, and the scarcity of malignant cases $(1 / 10,10 \%)$. In fact, in the case of PRMCa, recurrence rates of $40 \%$ and $55 \%$ were reported among 57 and 76 patients, respectively, in the two recent meta-analyses. ${ }^{2,3}$ Considering the lack of consensus on the appropriate management of PRMN recurrence, it is crucial to ensure that, based on pre-operative imaging findings, cysts with malignant characteristics are removed intact without decompression or rupture outside retrieval bags; this will reduce the risk of tumour dissemination. Further, the above-mentioned techniques, along with other procedures, including the use of wound-protectors, abdominal irrigation, and excision of trocar sites, may be helpful in minimising the risk of port-site metastasis, which is associated with poor outcomes in the case of malignant ovarian masses. $^{25}$

In summary, we present 10 cases of PRMN, including 1 case of PRMCa, 4 cases of bPRMC, and 5 cases of PRMC. This is the largest series of PRMNs dissected laparoscopically to date. Aside from the description of clinicopathological characteristics, which is in general accordance with previous knowledge, we provide detailed records and evaluation of imaging exams whose diagnostic roles have yet been fully explored. Although the results of our imaging analysis could only be presented through descriptive statistics due to the small number of cases, we found that certain radiological features, including larger size and lobulation of the cyst, presence and contrast enhancement of thick and irregular cyst walls, mural nodules, and internal septa, might be suggestive of malignancy; such cases deserve a more cautious approach. In addition, this study highlights the feasibility of laparoscopic resection with the help of retrieval bags or endobags for the management of PRMN in institutions with relevant experience. Prospective multi-centred studies are needed before any definitive conclusions can be drawn concerning the role of pre-operative imaging and the choice of surgical approach.

\section{Ethics and Consent}

All procedures performed in studies involving human participants were in accordance with the ethical standards of the Institutional Ethics Committee of Peking University First Hospital and with the 1964 Helsinki Declaration and its later amendments or comparable ethical standards. Written informed consent was provided by the patient in the typical case report to have the case details published. Written informed consent for the case series to be published was waived by the Institutional Ethics Committee of Peking University First Hospital owing to the retrospective nature of the study. The data presented were maintained with confidentiality and no identifying information of individual patient was included in this article.

\section{Acknowledgments}

The authors are grateful to the pathology department and statistical office of PKUFH. The authors would also like to thank Xin Ye, PhD, for his technical support.

\section{Author Contributions}

All authors made substantial contributions to conception and design, acquisition of data, or analysis and interpretation of data; took part in drafting the article or revising it critically for important intellectual content; gave final approval of the version to be published; and agree to be accountable for all aspects of the work.

\section{Funding}

The authors declare that there was no funding for the study.

\section{Disclosure}

The authors declare that they have no conflicts of interest in this work.

\section{References}

1. Dayan D, Abu-Abeid S, Klausner JM, Sagie B. Primary retroperitoneal mucinous cystic neoplasm: authors' experience and review of the literature. Am J Clin Oncol. 2016;39:433-440. doi:10.1097/COC. 0000000000000298

2. Wolf B, Kunert C, Horn LC, Einenkel J. Management of primary retroperitoneal mucinous tumors: a retrospective meta-analysis. Int J Gynecol Cancer. 2017;27(6):1064-1071. doi:10.1097/IGC.000 0000000001013

3. Myriokefalitaki E, Luqman I, Potdar N, Brown L, Steward W, Moss EL. Primary retroperitoneal mucinous cystadenocarcinoma (PRMCa): a systematic review of the literature and meta-analysis. Arch Gynecol Obstet. 2016;293(4):709-720. doi:10.1007/s00404015-3975-8

4. Handfield JR. Retroperitoneal cysts: their pathology, diagnosis and treatment. Br J Surg. 1924;12:119-134. doi:10.1002/bjs.1800124515

5. Roma AA, Malpica A. Primary retroperitoneal mucinous tumors: a clinicopathologic study of 18 cases. Am J Surg Pathol. 2009;33 (4):526-533. doi:10.1097/PAS.0b013e3181909018

6. Banerjee R, Gough J. Cystic mucinous tumours of the mesentery and retroperitoneum: report of three cases. Histopathology. 1988;12 (5):527-532. doi:10.1111/j.1365-2559.1988.tb01972.x

7. Motoyama T, Chida T, Fujiwara T, Watanabe H. Mucinous cystic tumor or retroperitoneum: a report of two cases. Acta Cytol. 1994;38 (2):261-266.

8. Tenti P, Carnevali L, Tateo S, Durola R. Primary mucinous cystoadenocarcinoma of the retroperitoneum: two cases. Gynecol Oncol. 1994;55(2):308-312. doi:10.1006/gyno.1994.1295 
9. Lee IW, Ching KC, Pang M, Ho TH. Two cases of primary retroperitoneal mucinous cystadenocarcinoma. Gynecol Oncol. 1996;63 (1):145-150. doi:10.1006/gyno.1996.0295

10. Song DE, Kim MJ, Khang SK, et al. Primary mucinous cystic neoplasm of the retroperitoneum: a report of three cases. Korean J Pathol. 2003;37:204-209.

11. Isse K, Harada K, Suzuki Y, et al. Retroperitoneal mucinous cystadenoma: report of two cases and review of the literature. Pathol Int. 2004;54(2):132-138. doi:10.1111/j.1440-1827.2004.01595.x

12. De Leon DC, Perez-Montiel D, Chanona-Vilchis J, et al. Primary retroperitoneal mucinous cystadenocarcinoma: report of two cases. World J Surg Oncol. 2007;5(1):5. doi:10.1186/1477-7819-5-5

13. Vargas AC, Lam V, P'ng CH. A rare case of primary retroperitoneal mucinous neoplasm in a male patient. Pathology. 2015;47 (4):384-386. doi:10.1097/PAT.0000000000000261

14. Yang DM, Jung DH, Kim H, et al. Retroperitoneal cystic masses: CT, clinical, and pathologic findings and literature review. Radiographics. 2004;24(5):1353-1365. doi:10.1148/rg.245045017

15. Riopel MA, Ronnett BM, Kurman RJ. Evaluation of diagnostic criteria and behavior of ovarian intestinal-type mucinous tumors: atypical proliferative (borderline) tumors and intraepithelial, microinvasive, invasive, and metastatic carcinomas. Am J Surg Pathol. 1999;23(6):617-635. doi:10.1097/00000478-199906000-00001

16. Hart WR. Mucinous tumors of the ovary: a review. Int $J$ Gynecol Pathol. 2005;24(1):4-25.

17. Tokai H, Nagata Y, Taniguchi $\mathrm{K}$, et al. The long-term survival in primary retroperitoneal mucinous cystadenocarcinoma: a case report. Surg Case Rep. 2017;3(1):117. doi:10.1186/s40792-017-0394-Z
18. Pesapane F, Van Renterghem S, Patella F, De Visschere P, Villeirs G. A case report and a literature review of primary retroperitoneal mucinous cystadenoma: the importance of imaging in diagnosis and management. Future Oncol. 2018;14(28):2923-2931. doi:10.2217/ fon-2017-0649

19. Lee SY, Han WC. Primary retroperitoneal mucinous cystadenoma. Ann Coloproctol. 2016;32(1):33-37. doi:10.3393/ac.2016.32.1.33

20. Nardi WS, Dezanzo P, Quildrian SD. Primary retroperitoneal mucinous cystadenoma. Int $J$ Surg Case Rep. 2017;39:218-220. doi:10.1016/j.ijscr.2017.08.029

21. Pellegrino B, Sikokis A, Bersanelli M, et al. Primary retroperitoneal cystoadenocarcinoma: a systematic review. Gynecol Obstet Invest. 2016;81(5):385-393. doi:10.1159/000446954

22. Ishigami K, Nishie A, Mochidome N, et al. Mucinous nonneoplastic cyst of the pancreas: CT and MRI appearances. Abdom Radiol (NY). 2017;42(12):2827-2834. doi:10.1007/s00261-017-1204-6

23. Lee JH, Byun JH, Kim JH, Lee SS, Kim HJ, Lee MG. Solid pancreatic tumors with unilocular cyst-like appearance on CT: differentiation from unilocular cystic tumors using CT. Korean J Radiol. 2014;15(6):704-711. doi:10.3348/kjr.2014.15.6.704

24. Nishino M, Hayakawa K, Minami M, et al. Primary retroperitoneal neoplasms: CT and MR imaging findings with anatomic and pathologic diagnostic clues. Radiographics. 2003;23(1):45-57. doi:10.1148/ rg.231025037

25. Huang KG, Wang CJ, Chang TC, et al. Management of port-site metastasis after laparoscopic surgery for ovarian cancer. $\mathrm{Am}$ J Obstet Gynecol. 2003;189(1):16-21. doi:10.1067/mob.2003.330

\section{Publish your work in this journal}

Cancer Management and Research is an international, peer-reviewed open access journal focusing on cancer research and the optimal use of preventative and integrated treatment interventions to achieve improved outcomes, enhanced survival and quality of life for the cancer patient.
The manuscript management system is completely online and includes a very quick and fair peer-review system, which is all easy to use. Visit http://www.dovepress.com/testimonials.php to read real quotes from published authors. 